\title{
Method for assessing the voltage stability limit during emergency outages of the line and generators in the power system
}

\author{
Nariman Rahmanov ${ }^{1}$, Victor Kurbatsky ${ }^{2}$, Huseyngulu Guliyev ${ }^{1}$, Nikita Tomin $^{2}$, Famil Ibrahimov ${ }^{1}$ \\ ${ }^{1}$ Azerbaijan Scientific-Research and Designed-Prospecting Institute of Energetics, Baku, Azerbaijan \\ ${ }^{2}$ Melentyev Energy Systems Institute Siberian Branch of the Russian Academy of Sciences, Irkutsk, Russia
}

\begin{abstract}
The paper proposes method and algorithm for obtaining the maximum transmitted power limit in the power system under the voltage stability conditions in case of emergency outages of its elements power lines and generating sets. The method is based on the analysis of the exaggerated states of the system created by increasing the values of the power sources (generators of power plants). The model of system states in extreme modes is described in the form of "extreme transmitted power - generating power values" relationships. The efficiency of the method is investigated on IEEE test schemes and the real Azerenergy system scheme. A comparison of the proposed method results with the evaluation of the critical conditions on the basis of trained neural network is presented.
\end{abstract}

\section{Introduction}

In recent years, the emergency outages of main elements happened in modern power systems generating units, power lines and transformers, as a result of limiting the possibilities of transit of large powers through transmission networks, lack of generation powers, corrosion of equipment and other factors. During frequent outages of these components, especially in maximum consumption conditions, the power system activity leads it to a state close to the voltage instability boundary, and the subsequent state can lead to a complete "blackout" of its most part.

Taking into account the existing state formed in the power system and frequent emergency outages of its components, there is a need to strengthen the requirements for voltage stability.

Other important factors leading to the formation of voltage stability are the electrical system configurations, the characteristics of generating sets and loads. With the aim of preserving the power system stability in the postemergency modes, subject to the cutoff of the main elements, the shunt capacitances (capacitor banks) and flexible transmission system (FACTS) installed in the "weak" nodes of the network are used [1-4].

The practical implementation of a large number of proposed methods for the determination of the static stability limit of the power grid leads to the analysis and control of successive exaggerating modes of electric networks up to maximum transmitting power. Usually, the exagerration is carried out by means of the control of the power generated by generators or the consumed power of load.

In paper [5] the linear and quadratic estimation of the network load (NL) for the predetermined system parameters, including the generator power values and loads in the nodes, is performed using the sensitivity analysis method. The searching of the node, in which the power value exaggeration from the normal to the critical state is possible, cannot be carried out by means of realization of this approach. The possibility of the power system mode exaggeration with the participation of all sources to the maximum power transmission in the network is considered in the paper.

The method of increase (expansion) of safety limits on the basis of exaggeration by means of the control of the power generated by the sources is proposed in the paper [6]. This method allows for obtaining the maximum generating power at the local point of the network.

The method of mode exaggeration to the optimal critical value using the source model is proposed in the paper under consideration. The proposed methodology allows for the determining the load limit in the form of generation power change dependence. This method makes it possible to reach the load limit of the network with the participation of all generating sources in the mode exagerration process.

\section{Methods of system mode exaggeration with the increase of the consumed power of load}

The essence of these methods is to identify the share of each source in the increase of the transmitted load and the losses of this power that occurred during transmission from the network. Let's assume that $K_{G i}$ is an indicator of increase of the generated active power of the generator, and $P_{G i, 0}$ is the basic (nominal) 
generated power of this source. In this case, the generation power $P_{G i}$ at the point, following the power increase, will be as follows:

$$
P_{G i}=P_{G i, 0}\left(1+K_{G i}\right)
$$

here for all the generators involved $i=1,2, \ldots, n$.

The value $K_{G i}$ of $i$ generator shows the degree of participation in the violation of the voltage stability limit. Analysis of existing methods allowing to establish a list of generators involved in the determination of voltage stability is given below.

Traditional approach. Usually in the system the generation, as in the case of planning in accordance with the existing rotary speed reserve, exceeds the predetermined value [7]. After the load power increase, the $i$ generator power can be represented as follows:

$$
\begin{gathered}
P_{G i}=P_{G i, 0}\left(1+K_{G i}\right)=P_{G i, 0}+\Delta P_{G i} \\
\sum_{N G} \Delta P_{G i}=\Delta P_{D}+\Delta P_{\text {loss }}
\end{gathered}
$$

where $P_{G i}$-generated power of generator $i ; P_{G i, 0}$ power of generator $i$ during rated load; $\Delta P_{G i}$-increase of power of generator $i ; \Delta P_{D}-$ load power increase; $\Delta P_{\text {loss }}-$ power loss increase; $N G$-number of generators.

Approach based on simulation of power distribution optimization. The traditional model of optimal power distribution can be formed taking into account the voltage stability criterion [8]. Minimization of the total costs for the generated power of generating units is as follows:

$$
A\left(P_{G i}\right)=\sum_{N G}\left(a_{G i} P_{G i}^{2}+b_{G i} P_{G i}+c_{G i}\right)
$$

In this case, the following condition must be met:

$$
\begin{gathered}
P_{G i}-(1+\lambda) P_{D i, 0}-\sum_{j=1}^{n}\left|U_{i}\right|\left|U_{j}\right|\left(G_{i j} \cos \delta_{i j}+B_{i j} \sin \delta_{i j}\right)=0 \\
Q_{G i}-(1+\lambda) Q_{D i, 0}-\sum_{j=1}^{n}\left|U_{i}\right|\left|U_{j}\right|\left(G_{i j} \sin \delta_{i j}-B_{i j} \cos \delta_{i j}\right)=0 \\
\left|P_{G i}\right|_{\min } \leq\left|P_{G i}\right| \leq\left|P_{G i}\right|_{\max } \\
\left|U_{i}\right|_{\min } \leq\left|U_{i}\right| \leq\left|U_{i}\right|_{\max } \\
S_{i j}=\sqrt{P_{i j}^{2}+Q_{i j}^{2}} \leq S_{i j, \max }
\end{gathered}
$$

where $a_{G i}, b_{G i}, c_{G i}$ - the coefficients of the "costs $-i$ unit power" dependence function; $P_{G i}, Q_{G i}$-active and reactive powers of the unit $i$ respectively; $P_{D i, 0}, Q_{D i, 0}$ - active and reactive powers of the power in the node $i$ respectively; $\left|U_{i}\right|_{\min },\left|U_{i}\right|_{\max }-$ lower and upper voltage limits in the node $i$ respectively; $\left|P_{G i}\right|_{\min },\left|P_{G i}\right|_{\max }$ - lower and upper generated voltage limits in the node $i$ respectively; $P_{i j}, Q_{i j}, S_{i j}$-active, reactive and total powers in $i j$ line respectively.
In this method, the exaggeration value is determined in the form of the difference between the optimal value and the originally defined values.

Method of selection of the boundary voltage value in electrical networks of the power system. This method allows for determining the maximum exaggeration value - load limit as a function of the active powers of generating units. The practical realization of this method can be carried out in the following three stages:

$1^{\text {st }}$ stage. The "load limit $P_{H \text { max }}$ - power increase value of each generator" dependence is determined. If the boundary load value is not taken into account as a basic, then it is determined by the $P-U$ curves constructed for the series of corresponding generators $P_{G i}$. The $P_{H \text { max }}\left(P_{G i}\right)$ graph is then approximated in the form of the following polynomial [9]:

$$
\begin{aligned}
P_{H M(j)}= & B_{j, 0}+B_{j, 1} P_{G, j}+\ldots+B_{j, n} P_{G, j}^{n}= \\
& =B_{j, 0}+\sum_{p=1}^{n} B_{j, p} P_{G, j}^{p}=B_{0}+P_{H m},_{j}
\end{aligned}
$$

where $P_{H M(j)}$-polynomial approximating the $P_{H M}$ curve in the case of two generators (basic generator and other generator associated with $j$ node); $B_{i j}$ - coefficients of approximating polynomial; $n$-number of coefficients; $P_{H M} 1$ - if the constant is not taken into account, then it is equal to $P_{H M}$.

$2^{\text {nd }}$ stage. The $P_{H \max }$ surface approximated for the multidimensional case (the case when more than 2 generating units participate in the maximization of the load limit of the network) was based on the division condition. In the case of participation of all units, the $P_{H \max }$ is determined by the following formula:

$$
P_{H \max }=B_{0}+\sum P_{H \max , j}
$$

Equation (11) describes the $P_{H \max }$ surface for all possible generators in the power system. Data for this can be obtained from combinations of all polynomial equations in the form of (10).

$3^{\text {rd }}$ stage. The last stage of the proposed method consists of determination of the best vector $P_{G, i}$, which gives a high value of the surface $P_{H \max }$. This value can be determined by means of maximization of the formula (11) within the following constraints:

$$
\sum P_{G j}=1, \quad 0 \leq P_{G j} \leq 1
$$

Studies at the first stage, consisting of determination of the dependence of the transmitted power limit $P_{\max }$ on the generated power value of each generator for the cases of the most probable emergency failures of individual elements of the system, are carried out by means of designed industrial program for the existing probability of power distribution. ETAP is used as such a program in the paper [10].

Thus, series of dependences of $P_{\max }^{j}$ on the power $P_{G i}^{j}$ of individual generator $i$ can be obtained for each case of emergency failure $" j "$ during the design experiments: 


$$
P_{\max }^{j}=F\left(P_{G i}^{j}\right)=b_{0}^{j}+\sum P_{\max }
$$

For each case of emergency failure, the global maximum of the quantity $P_{\max }^{j}$ reflecting the impact of the change of powers of all sources on $P_{\max }^{j}$ is determined by the extreme value of the function (12). For this purpose, one of the traditional methods can be used (for example, the Lagrangian method).

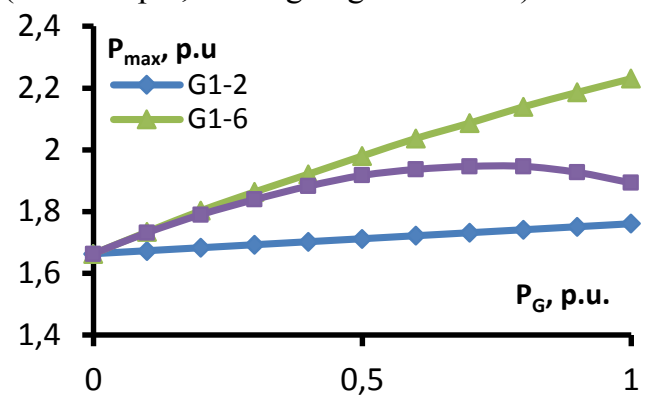

a)

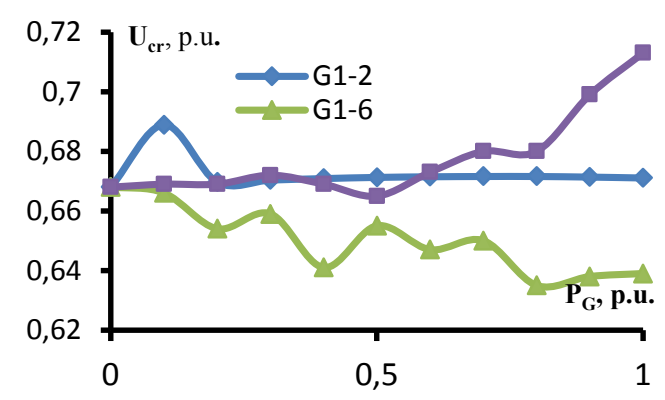

b)

Fig. 1. Curves of transmitted power limit (a) and critical voltage for option of 2 generators allocated for regulation

\section{Evaluation of the maximum system stability limit in different states of the network elements and generating unit}

In accordance with the proposed method, the limiting state of the system is estimated to reach the maximum of the $P-U$ curve at different values of the power increase and powers of generating sources. The size of the spatial state of the system in coordinates of the quantity of powers is determined by the number of sources selected for the operational dispatching control of the power system. The number of sources involved in the operational control of the 14-node IEEE test scheme is 4 . First, the effect on the voltage stability of 2 generators, then 3 generators and finally 4 generators is studied. The powers of sources connected to nodes 1 , 2, 3, 8 are $150,78,40$ and $40 \mathrm{MW}$, respectively. The total power consumption in the system is $260 \mathrm{MW}$.

When regulating during the simulation with the participation of two generators, the options of paired generators associated with the following buses were considered: 1 and 2 (G1-2), 1 and 6 (G1-6), 1 and 8 (G1-8). In all studied options the generator connected to bus 1 was accepted as balancing one.
The characteristic of the transmitted power limit change of line 1-2 is presented in the Fig.1,a. These curves were constructed on the basis of calculations for the voltage stability limit during the change of powers of generators $2,6,8$. It should be noted that the overall effect obtained from all pairs of generators is estimated as follows:

$$
P_{G, 1}+P_{G, 2}=1 ; P_{G, 1}+P_{G, 6}=1 ; P_{G, 1}+P_{G, 8}=1 ; \quad \mathrm{u}
$$

This suggests that, for example, if $20 \%$ is compensated by $\mathrm{G} 2$, then the remaining $80 \%$ is compensated by balancing source.

The graphs of critical voltage limit changes in the cases of the considered pair generators are represented in the Fig. 1,6.

As can be seen from Fig. 1, during various options of paired generators involved in the power regulation, the boundary parameters $\left(P_{\max }, U_{c r}\right)$ have different nature of change. Approximating relationships are presented in Table 1.

As can be seen from Fig.1, the maximum transmitted power limit value is formed in generators 6 and 8 respectively during the generation increase. For example, in $8^{\text {th }}$ generator the power increase only in the range of 0.6 (r. u.) can provide the maximum power limit in the network.

Table 1. Coefficients of approximating relationships

$$
\left(P_{\max }\right)_{i}=f\left(P_{G, i}\right)
$$

\begin{tabular}{|c|c|c|c|}
\hline Coefficients & $\mathbf{G 1 - 2} \mathbf{( j = 2 )}$ & $\begin{array}{c}\mathbf{G 1 - 6} \\
\mathbf{( j = 6}\end{array}$ & $\mathbf{G 1 - 8} \mathbf{( j = 8 )}$ \\
\hline$b_{j, 0}$ & 1,663 & 1,663 & 1,663 \\
\hline$b_{j, 1}$ & 0,0984 & 0,6518 & 0,7668 \\
\hline$b_{j, 2}$ & $-0,0011$ & 1,3101 & $-0,9968$ \\
\hline$b_{j, 3}$ & 0,000609 & $-9,5352$ & 2,3964 \\
\hline$b_{j, 4}$ & & 27,3342 & $-4,4472$ \\
\hline$b_{j, 5}$ & & $-39,3054$ & 3,1079 \\
\hline$b_{j, 6}$ & & 27,9063 & $-1,1972$ \\
\hline$b_{j, 7}$ & & $-7,7942$ & \\
\hline
\end{tabular}

The values of the coefficients of approximating polynomials, which are given in Table 1, represent the curves in Fig. 1 in the following form:

$$
\begin{gathered}
P_{\max (2)}\left(P_{G, 2}\right)=B_{0}+\sum_{p=1}^{3} B_{2, p} P_{G, 2}^{p} \\
P_{\max (6)}\left(P_{G, 6}\right)=B_{0}+\sum_{p=1}^{7} B_{6, p} P_{G, 6}^{p} \\
P_{\max (8)}\left(P_{G, 8}\right)=B_{0}+\sum_{p=1}^{6} B_{8, p} P_{G, 8}^{p}
\end{gathered}
$$

These polynomials are used to describe the spatial state surface of the system in the case of 3 and 4 generators involved for the subsequent control of the power balance mode in the system.

The curves of change of power limit and critical voltage in the system for the options of participation of paired generators $1-2,1-6,1-8$ in the regulation of general load transfer mode in the case of emergency 
failure of one of the lines (case N-1) are represented in the Fig.2.

In the case of pair generator $1-8$, the maximum transmitted power limit reaches $0,6 P_{\max (n-1)}$ at $P_{G, 8}=0,65$, and this indicates the decrease of the probable value of the transmitted power limit to $1,8 P_{\max } / 0,6 P_{\max (n-1)}=3$. Accordingly, during the failure of one of the lines in the system, the indicators, that determine the critical voltage value, deteriorate.

Equations approximating the curves in Fig.2, and their coefficients are presented below (Table 2).

$$
\begin{aligned}
& P_{\max (n-1)}^{(2)}=B_{0}+\sum_{p=1}^{7} B_{2, p} P_{G, 2}^{p} \\
& P_{\max (n-1)}^{(6)}=B_{0}+\sum_{p=1}^{6} B_{6, p} P_{G, 6}^{p} \\
& P_{\max (n-1)}^{(8)}=B_{0}+\sum_{p=1}^{8} B_{8, p} P_{G, 8}^{p}
\end{aligned}
$$

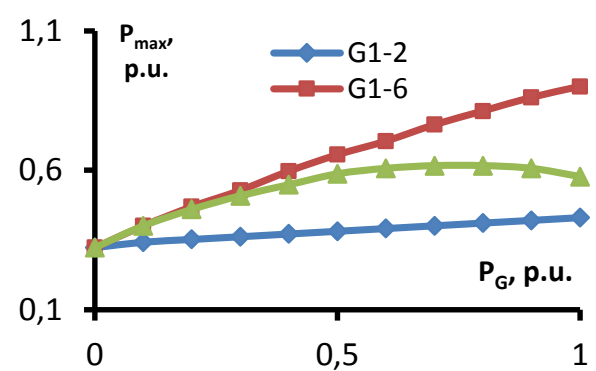

a)

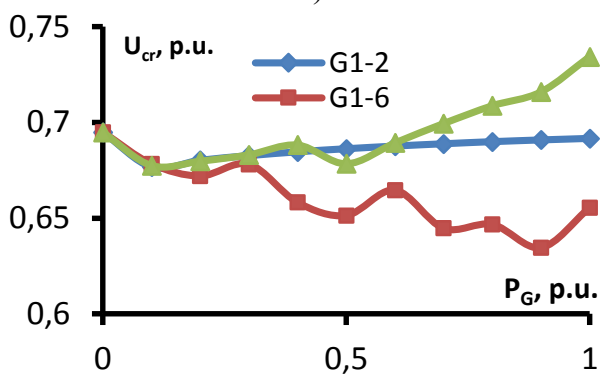

b)

Fig. 2. Change of the limiting state of the system by transmitted power value in the network in the case of regulating source 2 and emergency failure of one element $\mathrm{a}$ - transmitted power limit; b-critical voltage

Table 2. The values of coefficients of approximating relationships in the case of regulating source 2 and emergency failure of one element

\begin{tabular}{|c|c|c|c|}
\hline Coefficients & $\begin{array}{c}\text { G1-2 } \\
\mathbf{j}=\mathbf{2})\end{array}$ & $\begin{array}{c}\text { G1-6 } \\
\mathbf{j}=\mathbf{6})\end{array}$ & $\begin{array}{c}\mathbf{G 1 - 8} \\
\mathbf{( j = 8}\end{array}$ \\
\hline$b_{j, 0}$ & 0,3228 & 0,3228 & 0,3228 \\
\hline$b_{j, 1}$ & 0,3129 & 0,9175 & 0,9044 \\
\hline$b_{j, 2}$ & $-1,740$ & $-1,184$ & $-1,1935$ \\
\hline$b_{j, 3}$ & 6,9375 & 6,3475 & $-1,1513$ \\
\hline$b_{j, 4}$ & $-15,19$ & $-11,56$ & 12,9309 \\
\hline$b_{j, 5}$ & 18,461 & 10,086 & $-28,649$ \\
\hline$b_{j, 6}$ & $-11,69$ & $-3,362$ & 25,9782 \\
\hline$b_{j, 7}$ & 3,0103 & & $-8,5654$ \\
\hline
\end{tabular}

Case of three generators. Let's consider the option of three generators placed in nodes $1,2,6$ (G1-2-6); 1 , $2,8(\mathrm{G} 1-2-8)$ and $1,6,8(\mathrm{G} 1-6-8)$ respectively. The transmitted power limit on the network elements can be approximated by combination of the considered options for the case of two generators in all three options, showing the spatial limit state of the system in coordinates. For example, when considering G1-2-6 option, the $P_{\max }$ surface can be obtained by combining curves for generators 2 and 6 as follows:

$$
P_{\max }=B_{0}+\sum_{p=1}^{3} B_{2, p} P_{G, 2}^{p}+\sum_{p=1}^{5} B_{6, p} P_{G, 6}^{p}
$$

Similarly, the model can also be identified for options 1-2-8 and 1-6-8:

$$
\begin{aligned}
& P_{\text {max }}=B_{0}+\sum_{p=1}^{3} B_{2, p} P_{G, 2}^{p}+\sum_{p=1}^{6} B_{8, p} P_{G, 8}^{p} \\
& P_{\text {max }}=B_{0}+\sum_{p=1}^{5} B_{6, p} P_{G, 6}^{p}+\sum_{p=1}^{6} B_{8, p} P_{G, 8}^{p}
\end{aligned}
$$

It is possible to determine the powers of the generators $P_{G}$, allowing to estimate the maximum number $P_{\max }$ for each option from the sum total of considered generators, by optimizing the above equations. For example, for G1-2-6 option, the optimization process is described as follows:

$$
\max P_{\max }=B_{0}+\sum_{p=1}^{3} B_{2, p} P_{G, 2}^{p}+\sum_{p=1}^{5} B_{6, p} P_{G, 6}^{p}
$$

The conditions are as follows:

$$
\begin{gathered}
0 \leq P_{G, j} \leq 1 ; \quad j=2,6 \\
P_{G, 1}+P_{G, 2}+P_{G, 6}=1 ;
\end{gathered}
$$

Approximated curves and surfaces for the options G1-2-6, G1-2-8, G1-6-8, G1-2-6-8 obtained from equations (13) - (15) are shown in Fig.3. The spatial description, shown in Fig.4, is obtained by optimization and has the maximum coordinates $P_{\text {max }}$ of 0.2 and 0.8 , respectively, for the generators 2 and 8 .

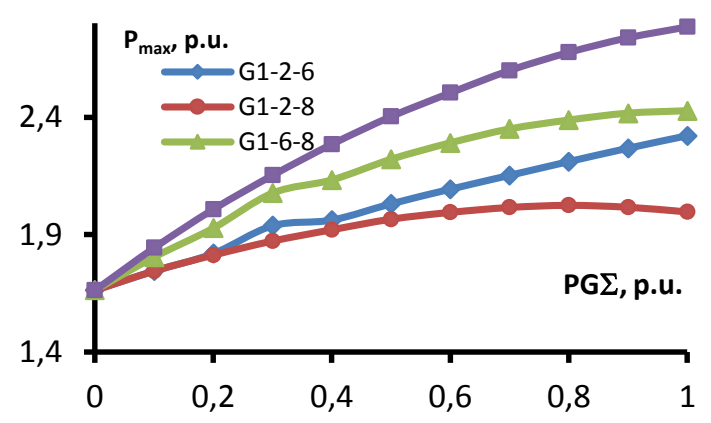




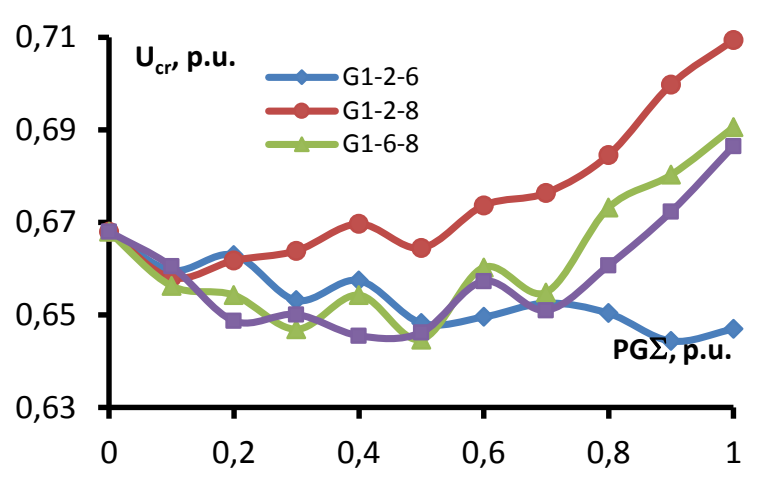

a)

Fig. 3. Curves of power limits (a) and critical voltage

(b) in cases of regulating sources 3 and 4

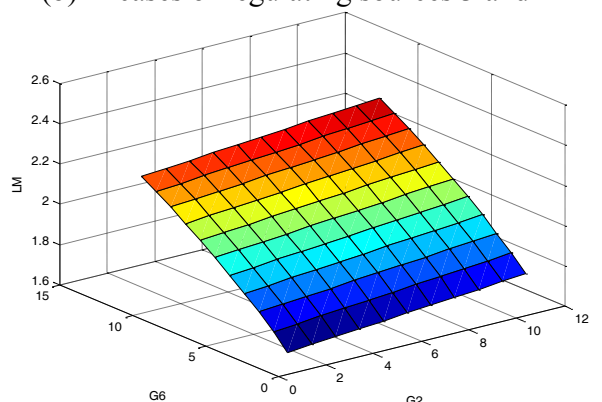

a)

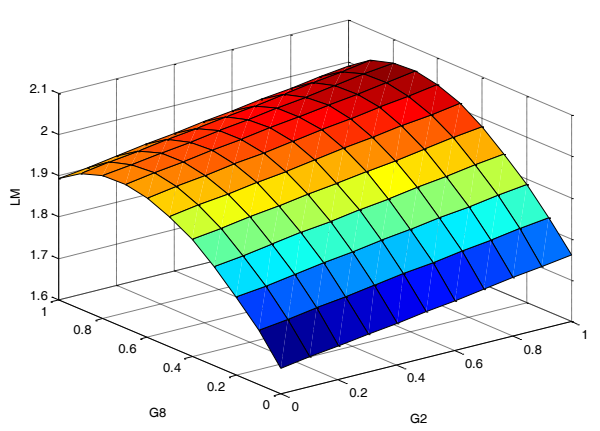

b)

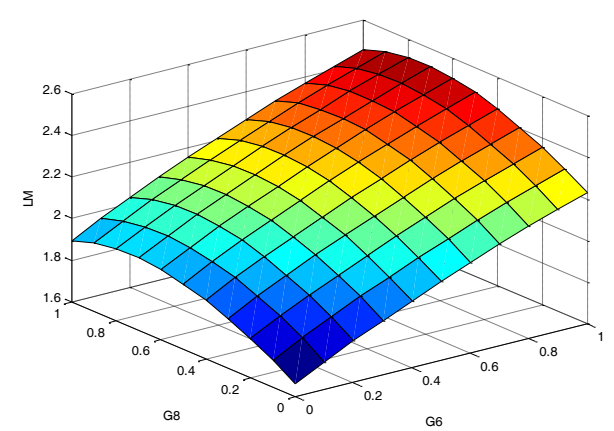

c)

Fig. 4. Surfaces of power limit for different cases a - case G1-2-6; b - case G1-2-8; c - case G1-6-8

This analysis was carried out on the basis of the results of computational experiments for the previously considered schemes and modes of the power system, but taking into account emergency failures of lines and sources. The curves and surfaces of the limit of power transmitted from the network in the case of options with different number of generators involved in power regulation are shown in the Fig.5-7.

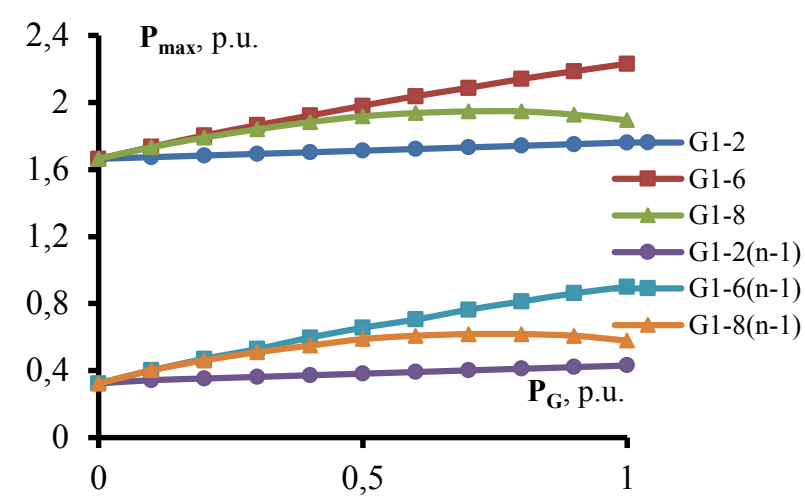

a)

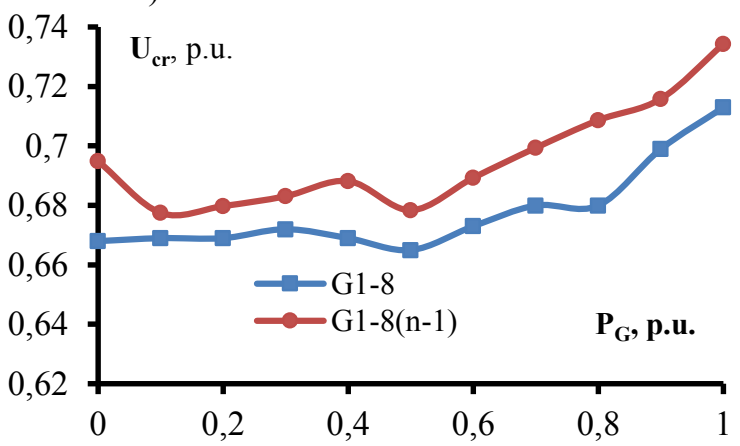

a)

Fig. 5. 2 Curves of power limits (a) and critical voltage (b) in cases of regulating source 2 and emergency failure of one element

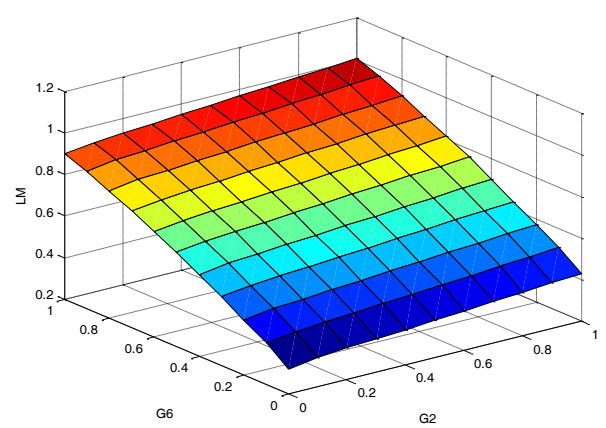

a)

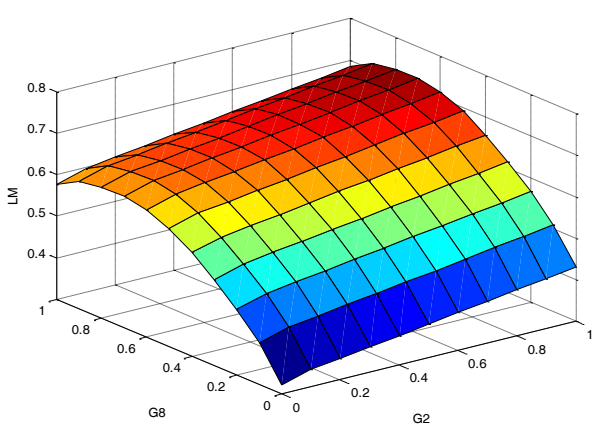

b) 


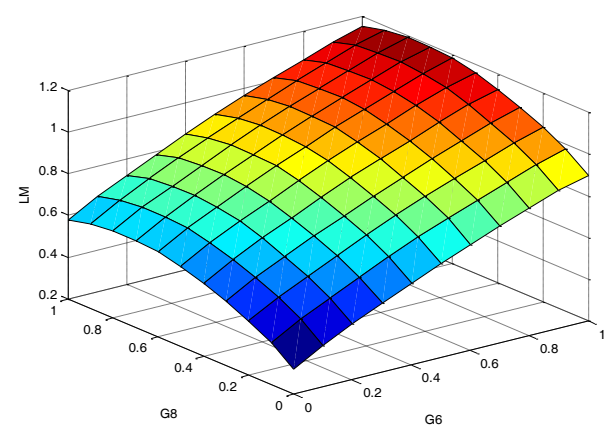

c)

Fig. 6. Surfaces of power limit transferred according to $\mathrm{N}-1$ criterion

a - case G1-2-6; b - case G1-2-8; c - case G1-6-8

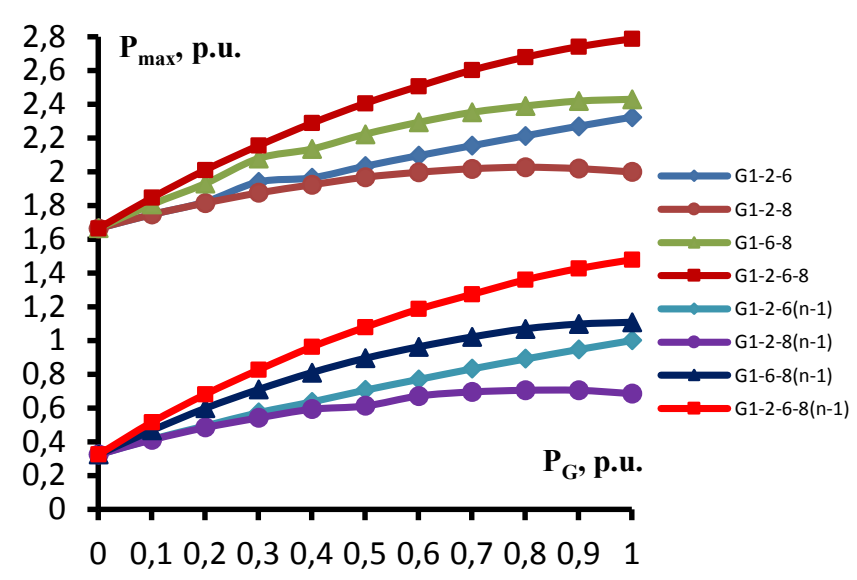

a)

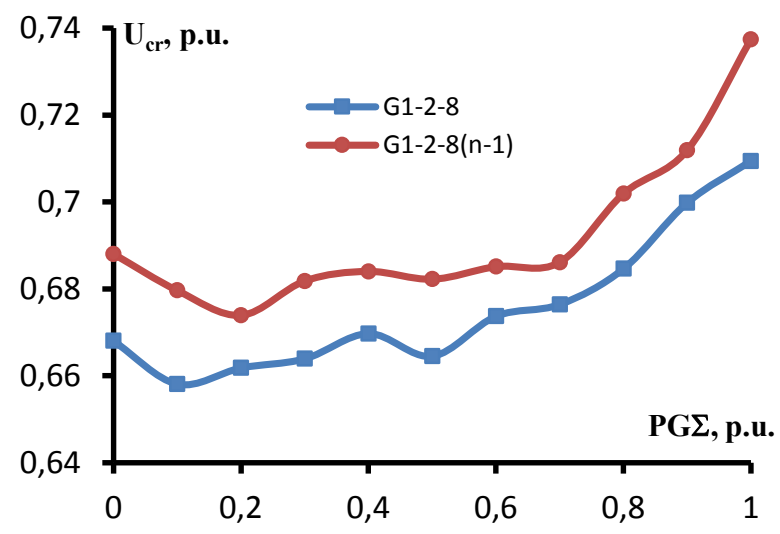

b)

Fig. 7. Curves of power limits (a) and critical voltage

(b) in cases of regulating sources 3 and 4 and emergency failure of one element

The results obtained for parameters $U_{c r, i}$ and $P_{\max , i}$ under conditions of different loads of generators and cases of action of one or more sources along with the base node in the system make possible to perform efficient measures for operational control of modes and determination of the most critical modes in different states of the power system.

\section{Conclusions}

1. The methods of increase of the consumed power of the load and the system mode exaggeration were investigated, these methods allow for determining the number and power of sources involved in the mode exaggeration, but can not be used in multi-machine systems.

2. Calculations were performed on standard schemes of the probable power distribution in cases of outage of lines and generators due to chance causes. On the basis of the calculation results, the critical voltage deviations in $U_{k r}$ nodes and the most critical nodes were determined under the condition of stability of the maximum power flows in the $P_{\max }$ lines. The effects of individual outages on critical quantities $U_{c r, i}$ and $P_{\max , i}$ were determined .

3. Critical quantities $U_{c r, i}$ and $P_{\max , i}$ were determined for the cases of different generator loads and the availability of one or more sources along with the backbone node in the system, as well as the dynamics of change. The obtained results allow for developing rational measures for operational control of modes and determination of the most critical rconditions in various states of the power system.

\section{References}

1. A complex of intelligent tools for the prevention of major accidents in power systems / Voropay N.I., Kurbatsky V.G., Tomin N.V. et al. Novosibirsk: Nauka, 2016, 332 p. ISBN 978-5-02-038717-1.

2. A. . Sode - Yome and N. Mithulananthan, "A maximum loading margin method for static voltage stability in Power Systems", IEEE Transactions on Power Systems, vol. 21., No. 2, may 2006. Pp. 799808.

3. N. Rahmanov., J. Bilbao, N. Tabatabayev, O. Kerimov, R. Rahmanov, "Probabilistic estimation of voltage stability limits in a power system with integrated distribution generation". Proceedings of the 12-th International Conference on Technical and Physical Problems of Electrical Engineering, 7-9 September 2016, Bilbao, Spain, pp. 5-11.

4. R. Shadmesgaran, A. Hashimov, N. Rahmanov "Optimal Location and capacity of parallel FACTS devices in order to improve static voltage stability and power losses reduction using genetic algorithm". Proceedings of the $12^{\text {th }}$ International Conference on Technical and Physical Problems of Electrical Engineering, 7-9 September 2016, Bilbao, Spain, 102106.

5. S. Grun, V. Dobson, and F. Alvarado "Sensitivity of loading margin to voltage collapse with respect to arbitrary parameters", pp. 362-372, Feb. 1997.

6. L. C. P. Da Silva, Y. Wang, V.F. da Costa, ana $\mathrm{W} . \mathrm{Xu}$, "Assesment of generator impact on system power transfer capability using modal participation factors", Proc. Inst. Elect. Eng., Generation, Transm., Distrib. Vol. 149 No-5., pp. 570-664. Sep. 2002. 
7. R. Wong and R.H. Lasseter, "Redispatching generation to increase power system security margin and support low voltage bus". IEEE Trans. Power Syst. Vol. 15., No -2., pp. 446-501, may 2000.

8. W.D. Rosehart, C.A. Canizares, V.H. Quintana, "Effect of detailed power system models in traditional and voltage stability constrained optimal power flow problems", IEEE Trans. Power Syst., vol. 18, No. 1, pp. 27-35, Feb. 2003.

9. A. Sade - Yome, and N. Mithulananthan, "Maximizing static voltage stability margin in power systems using a new generation pattern", in Proc. Anstralasion Universities Power Engineering Conf. Brisbane, Australia, Sep. 26-29. 2004.

10. ETAP, Power Station 4.0, User Guide. Volume II, Analysis of Modules. Operation Technology, Inc. Registered to ISO 9001, Certification No. A3147, 2002. 